\title{
Prevalence of multiple sclerosis in the L'Aquila district, central Italy
}

\author{
Rocco Totaro, Carmine Marini, Agostino Cialfi, Mario Giunta, Antonio Carolei
}

\begin{abstract}
Objective-To estimate the prevalence of multiple sclerosis in the L'Aquila district, central Italy.

Methods-All available case sources were screened. Definite and probable cases of multiple sclerosis, classified according to the Poser criteria, were considered as prevalent cases.

Results-On the prevalence day, 31 December 1996,158 patients ( 105 women and 53 men; ratio $2: 1$ ) affected by definite $(n=131)$ or probable $(n=27)$ multiple sclerosis were alive and resident in the L'Aquila district. Mean (SD) age was 38.4 (11.9) years (38.9 (11.7) years for women and $38.5(12.3)$ years for men, $p=0.9)$. The overall crude prevalence was $53.0 / 100000$ $(95 \%$ confidence interval $(95 \% \mathrm{CI})=45.4-$ $62.0) ; 68.4 / 100000(95 \% \mathrm{CI}=56.5-82.8)$ in women, and $36.7 / 100000(95 \% \mathrm{CI}=28.1-$ 48.0) in men. The prevalence was similar $(55.9 / 100000)$ when standardised to the 1996 European population. Mean (SD) age at onset of multiple sclerosis was $29.4(9.6)$ years and mean (SD) duration of the disease was $9.4(7.4)$ years, without any significant difference between sexes. Mean age at onset was significantly higher in patients with the primary progressive than in those with the relapsing-remitting course $(p=0.0002$, Scheffé's test $)$.

Conclusions-The prevalence found in the L'Aquila district gives support to the consideration of Italy as an area in which multiple sclerosis has been shown to have high prevalence at least in the populations that were surveyed recently.

(F Neurol Neurosurg Psychiatry 2000;68:349-352)
\end{abstract}

Keywords: multiple sclerosis; epidemiology; prevalence; Italy

Department of

Neurology, University

of L'Aquila, L'Aquila,

Italy

$\mathrm{R}$ Totaro

C Marini

A Cialfi

M Giunta

A Carolei

Correspondence to:

Professor Antonio Carolei,

Clinica Neurologica,

Dipartimento di Medicina

Interna e Sanità Pubblica,

Università degli Studi di

L'Aquila, 67100 L'Aquila,

Coppito, Italy

Received 30 March 1999 and in revised form

20 July 1999

Accepted 5 August 1999

In Europe, epidemiological data on multiple sclerosis showed increasing frequency at increasing latitudes. ${ }^{12}$ Variations in frequency within areas located at the same latitude were also reported. ${ }^{34}$ In Italy, previous epidemiological studies showed a low prevalence of multiple sclerosis, ranging between 4 and 21 cases per $100000,{ }^{5}$ whereas more recent studies found values between 39 and 102 cases per 100000 in different areas. ${ }^{5-12}$ All these studies were performed in northern and insular Italy, whereas no relevant large scale studies were conducted in central Italy.

To obtain reliable data on the impact of multiple sclerosis, we evaluated the prevalence of the disease in the L'Aquila district.
Patients and methods

The L'Aquila district is located in central Italy between latitudes $41^{\circ} 41^{\prime}$ and $42^{\circ} 31^{\prime} \mathrm{N}$, and covers an area of $5034.46 \mathrm{~km}^{2}$ (figure). The district is divided into 108 towns and has wide availability of health services and easy access to hospitals. At the 1991 census, the total study population, 48\% rural, was 297838 (153 535 women and 144303 men). ${ }^{13}$

Cases of multiple sclerosis were identified from all the available sources: clinical records of the departments of neurology; files of the neuroradiology services; records of the rehabilitation units; files of patients affiliated to the local section of the Italian Association for Multiple Sclerosis; records of the National Health Service; records of patients admitted within and out of the district; and general practitioners. To avoid the exclusion of patients with long latency between disease onset and diagnosis, all sources were continuously monitored after the prevalence day. Data on patients diagnosed with multiple sclerosis, or having clinical signs and symptoms of optic neuritis, myelopathy, and spastic paraparesis, were also reviewed. When the diagnosis was not fully supported by medical records, patients were asked to undergo further examinations. Date of onset, defined as the time of the first appearance of neurological signs and symptoms attributable to the disease, was obtained from the medical records or directly from the patient. All definite and probable cases of multiple sclerosis classified according to the Poser criteria ${ }^{14}$ were accepted as prevalent cases. Clinical course was defined as relapsing-remitting, primary progressive, or secondary progressive. ${ }^{15}$ Disability was coded by the expanded disability status scale (EDSS) ${ }^{16}$ and the European data base for multiple sclerosis (EDMUS) impairment scale (EIS).${ }^{17}$ All data were recorded on the EDMUS data base. ${ }^{17} 18$

Prevalence was computed considering all the 


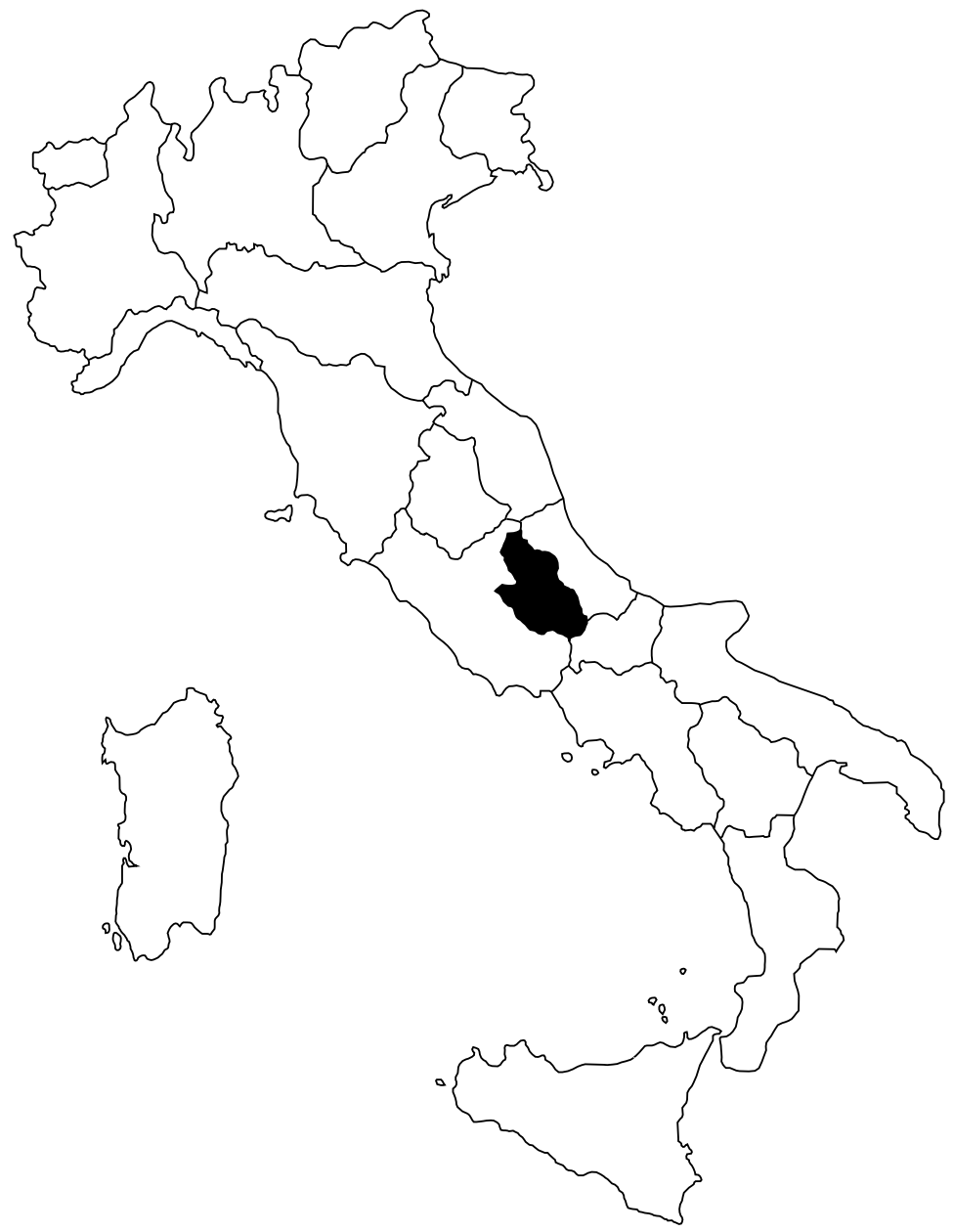

District of L'Aquila, central Italy. prevalence day, were also included into the study because the onset of the disease, after meticulous ascertainment, was found to have occurred previously (median 7 months). One hundred and twenty four patients $(78.5 \%)$ were identified from the neuroradiology services, $114(72.1 \%)$ from the departments of neurology, $87(55.1 \%)$ from the general practitioners, $39(24.7 \%)$ from the rehabilitation units, $25(15.8 \%)$ from the local section of the Italian Association for Multiple Sclerosis, 22 (13.9\%) from neurological departments out of the district, and $12(7.6 \%)$ from the Social Insurance Service (Istituto Nazionale della Previdenza Sociale). One hundred and twenty five patients $(79.1 \%)$ were identified by more than one source. Four patients $(2.5 \%)$ were estimated to be missed by the capturerecapture technique.

The overall crude prevalence was 53.0/100 000 (95\% CI 45.4-62.0); 68.4/100 000 (95\% CI 56.5-82.8) in women and 36.7/100 000 (95\% CI 28.1-48.0) in men. Age and sex specific prevalence are reported in table 1 . Prevalence was similar (55.9/100 000), when standardised for age and sex to the European population.

One hundred and five patients were women and 53 were men (ratio 2:1). Mean (SD) age was 38.4 (11.9) years (range, 12 to 73 years), without any significant difference $(p=0.9)$ between women (38.9 (11.7) years) and men (38.5 (12.3) years). Mean (SD) age at onset of multiple sclerosis was 29.4 (9.6) years (range 7 to 57 years), again without any significant difference $(p=0.7)$ between sexes (29.6 (10.1) years in women and 29.1 (8.9) years in men). The overall mean (SD) duration of the disease was 9.4 (7.4) years (range 0 to 33 years), 9.1 (7.1) years in women and 9.8 (8.2) years in men.

One hundred and thirty one patients $(83 \%)$ were affected by definite multiple sclerosis and $27(17.0 \%)$ by probable multiple sclerosis. The clinical course of the disease was relapsingremitting in 118 patients (74.7\%), secondary progressive in $29(18.3 \%)$, and primary progressive in $11(7.0 \%)$. Mean (SD) age at onset was 27.7 (8.3) years in patients with the relapsingremitting course, 32.8 (10.3) years in patients with the secondary progressive course, and 38.2 (14.3) years in patients with the primary progressive course. Mean age at onset was significantly higher in patients with the primary progressive than in those with the relapsingremitting course $(p=0.0002$, Scheffe's test). Symptoms at onset of the disease were fully diagnosed as having multiple sclerosis after the

Table 1 Age and sex specific prevalence rates of multiple sclerosis in the L'Aquila district on the prevalence day (31 December 1996)

\begin{tabular}{|c|c|c|c|c|c|c|c|c|c|}
\hline \multirow[b]{2}{*}{ Age (y) } & \multicolumn{3}{|c|}{ Men } & \multicolumn{3}{|c|}{ Women } & \multicolumn{3}{|l|}{ Total } \\
\hline & No & Rate $/ 10^{5}$ & $95 \% C I$ & No & Rate $/ 10^{5}$ & $95 \% C I$ & No & Rate $/ 10^{5}$ & $95 \% C I$ \\
\hline $0-14$ & 1 & 4.0 & $0.7-22.9$ & 1 & 4.2 & $0.7-23.9$ & 2 & 4.1 & $1.1-15.1$ \\
\hline $15-24$ & 7 & 33.1 & $16.0-68.3$ & 7 & 33.9 & $16.4-69.9$ & 14 & 33.5 & $19.9-56.2$ \\
\hline $25-34$ & 15 & 68.2 & $41.3-112.5$ & 38 & 173.7 & $126.6-238.3$ & 53 & 120.8 & $92.4-158.0$ \\
\hline $35-44$ & 11 & 53.0 & $29.6-94.8$ & 33 & 164.8 & $117.4-231.4$ & 44 & 107.9 & $80.4-144.8$ \\
\hline $45-54$ & 15 & 94.4 & $57.2-155.6$ & 14 & 87.3 & $52.0-146.5$ & 29 & 90.8 & $63.2-130.4$ \\
\hline $55-64$ & 2 & 12.0 & $3.3-43.8$ & 10 & 51.8 & $28.1-95.3$ & 12 & 33.4 & $19.1-58.3$ \\
\hline $65+$ & 2 & 8.7 & $2.4-31.6$ & 2 & 6.2 & $1.7-22.8$ & 4 & 7.3 & $2.8-18.7$ \\
\hline Total & 53 & 36.7 & $28.1-48.0$ & 105 & 68.4 & $56.5-82.8$ & 158 & 53.0 & $45.4-62.0$ \\
\hline
\end{tabular}


Table 2 Prevalence of multiple sclerosis in Italy

\begin{tabular}{|c|c|c|c|c|}
\hline Area & Year & $\begin{array}{l}\text { Rate per } \\
100000\end{array}$ & $95 \% C I$ & $\begin{array}{l}\text { Diagnostic } \\
\text { criteria }\end{array}$ \\
\hline Valle d'Aosta ${ }^{8}$ & 1985 & 38.4 & $28-51$ & a \\
\hline Pordenone $\mathrm{e}^{22}$ & 1987 & 51.5 & $34-75$ & $\mathrm{~b}$ \\
\hline Reggio Emilia and Modena ${ }^{9}$ & 1990 & 39.4 & $34-46$ & $\mathrm{c}$ \\
\hline Ferrara $^{6}$ & 1981 & 46.1 & $40-53$ & $\mathrm{~b}$ \\
\hline Ferrara $^{11}$ & 1993 & 68.3 & $60-77$ & $\mathrm{a}$ \\
\hline San Marino ${ }^{23}$ & 1982 & 51.6 & $26-92$ & $\mathrm{~b}$ \\
\hline Macerata $^{24}$ & 1988 & 37.5 & $31-45$ & $\mathrm{a}$ \\
\hline Ascoli Piceno $^{24}$ & 1988 & 42.8 & $36-50$ & $\mathrm{a}$ \\
\hline Pescara $^{25}$ & 1990 & 21.5 & $15-26$ & $\mathrm{a}$ \\
\hline Chieti $^{25}$ & 1990 & 22.0 & $17-27$ & $\mathrm{a}$ \\
\hline Northwestern Sardinia ${ }^{10}$ & 1991 & 102.6 & $92-115$ & $\mathrm{a}$ \\
\hline Caltanissetta ${ }^{7}$ & 1986 & 51.1 & $35-72$ & $\mathrm{a}$ \\
\hline Monreale ${ }^{12}$ & 1991 & 72.4 & $43-113$ & $\mathrm{a}$ \\
\hline Present study & 1996 & 53.0 & $45-62$ & $\mathrm{a}$ \\
\hline
\end{tabular}

$\mathrm{a}=$ Poser $e t a l,{ }^{14} \mathrm{~b}=$ Allison and Millar, ${ }^{26} \mathrm{c}=$ McAlpine et $a l .{ }^{27}$

recalled and reported by 146 patients (92.4\%). Motor symptoms were present in 63 patients $(43.1 \%)$, sensory in $62(42.4 \%)$, brainstem in 36 $(24.6 \%)$, and optic in $28(19.2 \%)$. In 81 patients $(51 \%)$ more than one symptom was reported. Median disability scores were 3.5 at the EDSS and 3.0 at the EIS; mean values were 3.9 at the EDSS and 3.7 at EIS.

\section{Discussion}

Our prevalence (53.3/100 000) was among the highest found in continental Italy (table 2) and was higher than that reported in studies conducted in the 1980 s using different diagnostic criteria. ${ }^{679222328}$ Surveys using comparable methods of case ascertainment reported lower prevalence in the Valle d'Aosta region, ${ }^{8}$ Marche region, ${ }^{24}$ and in the neighbouring districts of Pescara and Chieti. ${ }^{25} \mathrm{~A}$ higher prevalence was found only in the repeated prevalence surveys conducted in Ferrara ${ }^{11}$ and Monreale. $^{12}$

Recent studies reported a higher prevalence in surveys performed in the past, supporting the consideration of an increase in prevalence of multiple sclerosis in the past decades. It is very difficult to decide if this increased prevalence represents a true change or only reflects improved case identification and ascertainment, considering differences in methodology among the surveys. Further studies may help to map the prevalence of multiple sclerosis in Italy and might disclose future changes in prevalence, but are unlikely to resolve the question of whether or not estimates obtained before the introduction of new improved diagnostic methods were lower because of lower levels of detection or also because of lower prevalence of multiple sclerosis at that time.

The wide range of the latency between onset of symptoms and diagnosis might have limited the inclusion of patients who were still undiagnosed on the prevalence day. The continuous monitoring of all the available sources thereafter, helped to reduce this bias. Nevertheless, despite measures that should have contributed to minimise this occurrence, a minority of our patients $(4.4 \%)$ were diagnosed and included into the study after the prevalence day.

To avoid the exclusion of patients with multiple sclerosis who might have received medical care out of the district, we continuously monitored neighbouring hospitals. This additional measure allowed the identification and inclusion of $13.9 \%$ of our patients. Consequently, the proportion of cases $(2.5 \%)$ estimated to be missed by the capture-recapture technique was very low. Continuous clinical follow up and MRI contributed to the exclusion of patients with misdiagnosed disease who might have been included into the study. Finally, to avoid any related bias, we included only patients with definite and probable multiple sclerosis. Accordingly, the proportion of patients with probable multiple sclerosis $(17 \%)$ was within the range reported by other surveys (10.8 to $37.4 \%)^{11}{ }^{29-32}$

Our women to men ratio of $2: 1$ confirmed the well known higher occurrence of the disease in women. ${ }^{61129-32}$ As suggested by previous studies, mean age on the prevalence day, mean age at onset, and duration of the disease did not differ between sexes. ${ }^{611} 122132$ Mean age on the prevalence day (38.4 years) and mean duration of the disease (9.4 years) in our patients were in agreement with data reported in some studies $^{7233}$ and lower than in others. ${ }^{9} 1112293132$ This difference might have depended on the exclusion of some patients with a remote occurrence of symptoms of multiple sclerosis. In fact, before the introduction of MRI to screen patients with multiple sclerosis, the possibility of diagnosing the disease in the presence of a very benign course was unlikely. It is also recognised that patients with a benign course did not often seek medical advice. Moreover, mean age on the prevalence day and duration of the disease is reported to increase when surveys were replicated in the same area. ${ }^{671112}$

We confirmed the significantly higher mean age at onset in patients with the primary progressive than in those with the relapsingremitting course ${ }^{11} 1534$ in the presence of widely reported differences. ${ }^{34-36}$ Whether these differences were so important to suggest different diseases or represented different aspects of the same disease remains to be clarified. ${ }^{36}$ Among our patients there was a predominance of motor and sensory disturbances with respect to optic neuritis and brainstem symptoms at onset, as already reported by others. ${ }^{15} 29$

Our findings support the consideration of Italy as an area in which multiple sclerosis has a high prevalence, in agreement with the geographical zonal division in high, medium, and low frequency. ${ }^{2}$ However, it is worth mentioning that recently reported estimates from North America and Europe >100/100 000 might alternatively suggest considering Italy as a medium prevalence zone. ${ }^{37}$

This study was supported in part by a grant from the Istituto Superiore di Sanità-Secondo progetto di ricerca sulla Sclerosi Superiore di Sanita- Secondo progetto di ricerca sulla Sclerosi
Multipla (96/J/T42). We thank Professor Christian Confavreux Multipla (96/J/T42). We thank Professor Christian Confavreux
for his helpful comments and Professor Domenico Gambi and for his helpful comments and Professor Domenico Gambi and
Doctor Carlo Pozzilli for their contribution to the identification of patients.

1 Kurtzke JF. Epidemiologic contributions to multiple sclerosis: an overview. Neurology 1980;30:61-79.

2 Kurtzke JF. MS epidemiology world wide. One view of current status. Acta Neurol Scand 1995;(suppl 161):23-33.

3 Rosati G. Descriptive epidemiology of multiple sclerosis in Europe in the 1980s: a critical overview. Ann Neurol 1994; 36(suppl 2):164-74. 
4 Granieri E, Casetta I, Tola MR. Epidemiology of multiple sclerosis in Italy and southern Europe. Acta Neurol Scand sclerosis in Italy and sou

5 Rosati G. Descriptive epidemiology of multiple sclerosis in Italy. Rivista de Neurologica 1987;57:5-12.

6 Granieri E, Tola R, Paolino E, et al. The frequency of multiple sclerosis in Italy: a descriptive study in Ferrara. Ann Neurol 1985;17:80-4.

7 Savettieri G, Elian M, Giordano D, et al. A further study on the prevalence of multiple sclerosis in Sicily: Caltanissetta city. Acta Neurol Scand 1986;73:71-5.

8 Sironi L, Mamoli A, D'Alessandro G, et al. Frequency of multiple sclerosis in Valle d'Aosta, 1971-85. Neuroepidemiology 1991;10:66-90.

9 Guidetti D, Cavalletti S, Merelli E, et al. Epidemiological survey of multiple sclerosis in the provinces of Reggio Emilia and Modena, Italy. Neuroepidemiology 1995;14:713.

10 Rosati G, Aiello I, Pirastru MI, et al. Epidemiology of multiple sclerosis in northwestern Sardinia: further evidence for ple sclerosis in northwestern Sardinia: further evidence for higher frequency in Sardinians con
Neuroepidemiology 1996;15:10-19.

11 Granieri E, Malagù S, Casetta I, et al. Multiple sclerosis in Italy. A reappraisal of incidence and prevalence in Ferrara. Italy. A reappraisal of incide
Arch Neurol 1996;53:793-8.

12 Savettieri G, Salemi G, Ragonese P, et al. Prevalence and incidence of multiple sclerosis in the city of Monreale, Italy. 7 Neurol 1998;245:40-3.

13 Istituto Centrale di Statistica. Popolazione e abitazioni. $13^{\circ}$ censimento generale della popolazione e delle abitazioni. 20 ottobre 1991. Fascicolo generale 66, L'Aquila, Roma: Istituto Poligraphio e Zecca della Stato, 1993:1-300.

14 Poser CM, Paty DW, Scheinberg L, et al. New diagnostic criteria for multiple sclerosis: guidelines for research protocols. Ann Neurol 1983;13:227-31.

15 Lublin FD, Reingold SC. Defining the clinical course of multiple sclerosis: results of an international survey. Neurology 1996;46:907-11.

16 Kurtzke JF. Rating neurologic impairment in multiple Kurtzke $J \mathrm{~F}$. Rating neurologic impairment in multiple sclerosis: an expanded disat
rology $1983 ; 33: 1444-52$.

17 Confavreux C, Compston DAS, Hommes OR, et al. EDMUS, a European database for multiple sclerosis. $\mathscr{f}$ Neurol Neurosurg Psychiatry 1992:55:671-6.

18 Confavreux C, Aimard G, Devic M. Course and prognosis of multiple sclerosis assessed by the computerized data processing of 349 patients. Brain 1980;103:281-300

19 Rothman KJ, Greenland S. Modern epidemiology. Philadelphia: Lippincott-Raven, 1998.

20 EUROSTAT. Demographic statistics, 1997: population and social conditions. Luxemburg: Office for Official Publications of the European Communities, 1997:1-275. (Yearbooks and Yearly Statistics 3A.)
21 McCarty DJ, Tull ES, Moy CS, et al. Ascertainment McCarty DJ, Tull ES, Moy CS, et al. Ascertainment corrected rates: applications of capt

22 Diodato S, Cattaino G, Lisotto C, et al. Multiple sclerosis in epidemiological survey in Pordenone. In: Battaglia MA, Crimi G, eds. An update on multiple sclerosis. Bologna, Monduzzi, 1989:231-3.

23 Morganti G, Naccarato S, Elian M, et al. Multiple sclerosis in the Republic of San Marino. F Epidemiol Community Health 1984;38:23-8.

24 Angeleri F, Bollettini G, Brizioli E, et al. A prevalence study of multiple sclerosis in the Regione Marche, Italy. In: Battaglia MA, Crimi G, eds. An update on multiple sclerosis. Bologna, Monduzzi, 1989:209-12.

25 Malatesta G, Gabriele A, Macor S, et al. Diffusion of multiple sclerosis in two Italian provinces, Chieti-Pescara. Ital f Neurol Sci 1991;12(suppl 5):83.

26 Allison RS, Millar JHD. Prevalence and familial incidence of disseminated sclerosis. A report to the Northern Ireland Hospital authority on the results of a three-year survey. Ulster Med f 1954;23(suppl 2):5-27.

27 McAlpine D, Lunsden CE, Acheson ED. Multiple sclerosis. A reappraisal. Edinburgh, Churchill-Livingstone, 1976:101-3

28 Salerni E, D'Aurizio C, D'Andrea F, et al. A prevalence study of multiple sclerosis in L'Aquila, central Italy. Clin Neurol Neurosurg 1988;90:112-16.

29 McDonnell GV, Hawkins SA. An epidemiologic study of multiple sclerosis in Northern Ireland. Neurology 1998;50: 423-8.

30 Midgard R, Riise T, Nyland H. Epidemiologic trend in multiple sclerosis in Møre and Romsdal, Norway: a prevalence/ incidence study in a stable population. Neurology 1991;41: incidence

31 Ford HL, Gerry E, Airey CM, et al. The prevalence of multiple sclerosis in the Leeds Health Authority. $\mathcal{F}$ Neurol Neurosurg Psychiatry 1998;64:605-10.

32 Shepherd DI, Summers A. Prevalence of multiple sclerosis in Rochdale. F Neurol Neurosurg Psychiatry 1996;61:415-17

33 Benito-Léon J, Martín E, Vela L, et al. Multiple sclerosis in Móstoles, central Spain. Acta Neurol Scand 1998;98:238-

$34 \mathrm{McD}$ onnell GV, Hawkins SA. Clinical study of primary progressive multiple sclerosis in Northern Ireland, UK. 7 Neurol Neurosurg Psychiatry 1998;64:451-4.

35 Hillert J, Grønning M, Nyland, et al. An immunogenetic heterogeneity in multiple sclerosis. F Neurol Neurosurg Psychiatry 1992;55:887-90.

36 Thompson AJ, Polman CH, Miller DH, et al. Primary progressive multiple sclerosis. Brain 1997;120:1085-96.

37 Weinshenker BG. Epidemiology of multiple sclerosis. Neurol Clin 1996;14:291-308. 